\title{
THE RADICAL OF THE CENTER OF A GROUP ALGEBRA
}

\author{
D. S. PASSMAN
}

\begin{abstract}
Let $K[G]$ denote the group algebra of a finite group $G$ over a field $K$ of characteristic $p>0$ and let $\mathscr{Z}=\mathbf{Z}(K[G])$. In this paper, we offer a bound for the nilpotence degree of the Jacobson radical $J \mathscr{Z}$ in terms of the order of a Sylow $p$-subgroup of $G$.
\end{abstract}

Let $K[G]$ denote the group algebra of the finite group $G$ over the field $K$. If $|G| \neq 0$ in $K$, then by Maschke's theorem [1, Theorem 2.4.2], $K[G]$ and $\mathscr{Z}=$ $\mathbf{Z}(K[G])$ are both semisimple. On the other hand, if $|G|=0$ in $K$, then $\hat{G}=\Sigma_{x \in G} x$ is a nonzero central element of $K[G]$ of square zero and hence both $K[G]$ and $\mathscr{Z}$ are not semisimple. In this paper, we study the Jacobson radical of $\mathcal{Z}$ and we bound its nilpotence degree. In view of the above remarks, we can assume that char $K=p>0$. The main result is then:

THeOREM. Let char $K=p>0$ and let $|G|=p^{a} b$ with $p \nmid b$. If $J \mathscr{Z}$ denotes the Jacobson radical of $\mathscr{Z}=\mathbf{Z}(K[G])$, then

$$
(J \mathscr{Z})^{\left(p^{a+1}-1\right) /(p-1)}=0 .
$$

The proof of this is based on ideas of W. Willems and in particular on [2, Hilfsatz 4.3]. However here we work totally within $K[G]$ rather than dealing with central characters in characteristic zero.

We start by listing notation and a few basic facts (see [1, pp. 138-141]). Let $D$ be a $p$-subgroup of $G$.

(1) $G(D)$ is the span of all class sums of $G$ with defect group $\subseteq_{G} D$. By [1, Lemma 4.3.12], $G(D)$ is an ideal of $\mathcal{Z}$.

(2) $\tilde{g}(D)$ is the span of all class sums of $G$ with defect group $<_{G} D$. It follows from the above that $\tilde{g}(D)$ is also an ideal of $\mathcal{Z}$ which is clearly contained in $g(D)$.

(3) $\pi_{\mathbf{C}(D)}$ is the natural projection map $\pi_{\mathbf{C}(D)}: K[G] \rightarrow K[\mathbf{C}(D)]$. By [1, Theorem 4.3.10], $\pi_{C(D)}$ induces a homomorphism, the Brauer homomorphism, from $\mathbf{Z}(K[G])$ to $\mathbf{Z}(K[\mathbf{C}(D)])$.

(4) If $g \in G$, we write $g=g_{p^{\prime}} g_{p}$ as the product of its $p^{\prime}$ - and $p$-parts. We use $\sim$ to denote conjugates in $G$ and we use $d(\cdots)$ to denote defect groups. Furthermore $\omega(K[D])$ is the augmentation ideal of $K[D]$.

Received by the editors December 8, 1978.

AMS (MOS) subject classifications (1970). Primary $20 \mathrm{C05.}$ 
LemMa 1 . Let $D$ be a central p-subgroup of $G$. If $g, h \in G$ we write $g \approx h$ if and only if $g D \sim h D$, that is, if and only if these elements are conjugate in $G / D$.

(i) $\approx$ is an equivalence relation and if $g \approx h$ then $d(g) \sim d(h)$.

(ii) Let $g \in G$ with $d(g)=D$ and let $p^{n} \geqslant|G|$. Then $h^{p^{n}} \sim g^{p^{n}}$ if and only if $h \approx g$.

(iii) Let $\beta \in K[G]$ be nilpotent with $\beta=\Sigma b_{x} x$. If $d(g)=D$, then $\Sigma_{h \approx g} b_{h}=0$.

Proof. (i) It is clear that $\approx$ is an equivalence relation. Furthermore, since $D$ is central, $g \approx h$ implies that $\mathbf{C}(g) \sim \mathbf{C}(h)$ and hence $d(g) \sim d(h)$.

(ii) Let $d(g)=D$. Then $g_{p}$ is a $p$-element commuting with $g$ so $g_{p} \in D \subseteq \mathbf{Z}(G)$. Thus $\mathbf{C}\left(g_{p^{\prime}}\right)=\mathbf{C}(g)$ and $d\left(g_{p^{\prime}}\right)=D$. Now suppose $h^{p^{n}} \sim g^{p^{n}}$. Then since $p^{n} \geqslant|G|$ we have

$$
\left(h_{p^{\prime}}\right)^{p^{n}}=h^{p^{n}} \sim g^{p^{n}}=\left(g_{p^{\prime}}\right)^{p^{n}}
$$

so $h_{p^{\prime}} \sim g_{p^{\prime}}$ and hence $d\left(h_{p^{\prime}}\right) \sim d\left(g_{p^{\prime}}\right)=D$. But $h_{p}$ and $g_{p}$ are $p$-elements commuting with $h_{p^{\prime}}$ and $g_{p^{\prime}}$ respectively so $h_{p}, g_{p} \in D \triangleleft G$ and $h \approx h_{p^{\prime}} \approx g_{p^{\prime}} \approx g$. Conversely if $h \approx g$, then $h \sim g d$ for some $d \in D \subseteq \mathbf{Z}(G)$ and $h^{p^{n}} \sim g^{p^{n}} d^{p^{n}}=g^{p^{n}}$.

(iii) Let $\beta \in K[G]$ be nilpotent and let $d(g)=D$. Choose $p^{n}$ so that $p^{n}>|G|$ and $\beta^{p^{n}}=0$. If $\beta=\sum b_{x} x$, then by [1, Lemma 2.3.1]

$$
0=\beta^{p^{n}}=\sum b_{x}^{p^{n}} x^{p^{n}}+\gamma
$$

for some $\gamma \in[K[G], K[G]]$. By [1, Lemma 2.3.2] the sum of the coefficients in $\gamma$ of terms conjugate to $g^{p^{n}}$ is zero. Hence we have

$$
0=\sum_{h^{p^{n}} \sim g^{p^{n}}} b_{h}^{p^{n}}=\left(\sum_{h^{p^{n}} \sim g^{p^{n}}} b_{h}\right)^{p^{n}} .
$$

But by (ii) above, $h^{p^{n}} \sim g^{p^{n}}$ if and only if $h \approx g$, so we obtain $\sum_{h \approx g} b_{h}=0$.

LEMMA 2. Let $D$ be a central $p$-subgroup with $|D|=p^{d}$. Then

(i) $\mathscr{G}(D) \cdot J \mathscr{Z}=\omega(K[D]) \cdot I(D)$,

(ii) $\mathscr{G}(D) \cdot(J \mathscr{Z})^{p^{d}}=0$.

Proof. (i) Observe that $\omega(K[D]) \subseteq \mathscr{Z}$ and that $\omega(K[D])$ is nilpotent, by

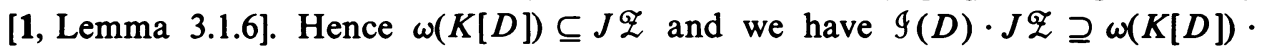
$g(D)$.

We now consider the reverse inclusion and we use the notation of the preceding lemma. Let $y \in G$ with $d(y)=D$ and let $\alpha \in J \mathscr{Z}$. Then $\alpha$ is nilpotent and central so $\beta=y \alpha$ is nilpotent. Write

$$
\beta=\beta_{1}+\beta_{2}+\cdots+\beta_{s}+\gamma
$$

where each $\beta_{j}$ is the sum of those terms in $\beta$ belonging to a single equivalence class under $\approx$ and with elements having defect group $D$. Furthermore $\gamma$ is the sum of the remaining terms where all defect groups are strictly larger than $D$, since $D$ is central.

Fix coset representatives $z_{1}, z_{2}, \ldots, z_{k}$ for $D$ in $G$ and for any $\delta \in K[G]$ define $\delta^{*}=\sum_{i} \delta^{z_{i}}$. Then, since $D$ is central, $\delta^{*}$ is independent of the choice of representatives $z_{i}$ and hence $\delta^{*} \in \mathscr{Z}$. Clearly 


$$
\beta^{*}=\beta_{1}^{*}+\beta_{2}^{*}+\cdots+\beta_{s}^{*}+\gamma^{*} .
$$

Note that $\alpha$ is central and $\beta=y \alpha$ so

$$
\beta^{*}=y^{*} \alpha=[\mathbf{C}(y): D] \hat{\hat{f}}_{y} \alpha
$$

where $\hat{\mathfrak{f}}_{y}$ denotes the class sum of $y$. Since $d(y)=D$ and $G(D)$ is an ideal of $\mathscr{Z}$ we have $\beta^{*} \in \mathscr{G}(D)$.

Consider $\beta_{j}$. By Lemma 1(iii), we can write $\beta_{j}=\Sigma c_{u} u$ where all $u \approx g_{j}$, for some fixed $g_{j}$, and where $\Sigma c_{u}=0$. Thus $\beta_{j}^{*}=\Sigma c_{u} u^{*}$. Now, since $D$ is central, the condition $u \approx g_{j}$ yields easily

$$
u^{*}=d_{u} g_{j}^{*}=d_{u}\left[\mathbf{C}\left(g_{j}\right): D\right] \hat{\mathfrak{f}}_{g_{j}}
$$

for some element $d_{u} \in D$. Thus

$$
\beta_{j}^{*}=\sum c_{u} u^{*}=\left(\sum c_{u} d_{u}\right)\left[\mathbf{C}\left(g_{j}\right): D\right]_{\mathrm{f}_{j}} \in \omega(K[D]) \cdot \mathscr{G}(D) \subseteq \mathscr{G}(D)
$$

since $\Sigma c_{u}=0$ and $d\left(g_{j}\right)=D$, by definition of $\beta_{j}$.

Now $\beta^{*}=\beta_{1}^{*}+\cdots+\beta_{s}^{*}+\gamma^{*}$ and we know that $\beta^{*}, \beta_{j}^{*} \in \mathscr{G}(D)$. Hence $\gamma^{*} \in \mathscr{G}(D)$. But $\gamma$ involves only group elements with defect group strictly larger than $D$, so this yields $\gamma^{*}=0$. Hence

$$
[C(y): D] \hat{\hat{f}}_{y} \alpha=\beta^{*}=\beta_{1}^{*}+\beta_{2}^{*}+\cdots+\beta_{s}^{*} \in \omega(K[D]) \cdot g(D)
$$

and, since $[C(y): D]$ is a $p^{\prime}$-number, we have $\hat{\mathfrak{f}}_{y} \alpha \in \omega(K[D]) \cdot g(D)$. This clearly proves part (i).

(ii) Since $g(D) \cdot J \mathscr{Z}=\omega(K[D]) \cdot g(D)$, it follows easily by induction that $g(D) \cdot(J \mathscr{Z})^{i}=\omega(K[D])^{i} \cdot g(D)$. Since $\omega(K[D])^{p^{d}}=0$, the result follows.

LEMMA 3. Let $D$ be any $p$-subgroup of $G$ with $|D|=p^{d}$. Then

(i) $\mathscr{g}(D) \cdot(J \mathscr{Z})^{p^{d}} \subseteq \tilde{G}(D)$,

(ii) $\mathscr{G}(D) \cdot(J \mathscr{Z})^{\left(p^{\frac{d}{d+1}}-1\right) /(p-1)}=0$.

Proof. (i) Since $G(D)$ is an ideal of $\mathscr{Z}$, we have $\mathscr{G}(D) \cdot(J \mathscr{Z})^{p^{d}} \subseteq g(D)$. Thus we need only show that the left-hand term has no class sums with defect group $D$. But observe that if $\hat{\mathfrak{t}}$ is a class sum with defect group $D$, then $\pi(\hat{\mathfrak{t}}) \neq 0$ where $\pi=\pi_{C(D)}$ denotes the Brauer homomorphism from $\mathscr{Z}$ to $\mathbf{Z}(K[\mathbf{C}(D)])$. Thus it clearly suffices to show that

$$
\pi(G(D)) \cdot \pi(J \mathscr{Z})^{p^{d}}=0 .
$$

Let $C=\mathbf{C}(D)$. Then by definition, $\pi(\mathscr{L}) \subseteq \overline{\mathcal{Z}}=\mathbf{Z}(K[C])$, and, since nilpotent elements map to nilpotent elements, we have $\pi(J \mathscr{Z}) \subseteq J \overline{\mathcal{L}}$. Now let $g$ be a support element in $\pi(g(D))$. Since $g \in C$ and $\hat{\mathfrak{f}}_{g} \in \mathscr{G}(D)$, it follows that $D$ is a Sylow $p$-subgroup of $\mathbf{C}_{G}(g)$. Furthermore, if $D_{1}$ is a Sylow $p$-subgroup of $\mathbf{C}_{C}(g)=C \cap$ $\mathrm{C}_{G}(g)$, then, since $D_{1} \subseteq C$, we see that $D_{1} D$ is a $p$-subgroup of $\mathrm{C}_{G}(g)$ so $D_{1} D=D$ and $D_{1} \subseteq D \cap C=\bar{D}$, the center of $D$. We conclude from all of this that

$$
\pi(g(D)) \subseteq 9_{C}(\bar{D})
$$

where $g_{C}(\bar{D})$ denotes the span of all class sums in $K[C]$ with defect group contained in $\bar{D}$, a central $p$-subgroup of $C$. Thus 


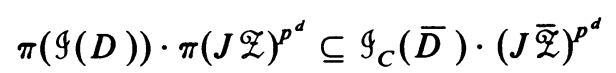

and the latter is zero, by Lemma 2(ii), since $\bar{D}$ is central in $C$ and $|\bar{D}|<|D|=p^{d}$. In view of our previous remarks, this proves (i).

(ii) We proceed by induction on $d$. The case $d=0$ follows from Lemma 2(ii). If $D \neq\langle 1\rangle$, then

$$
\tilde{g}(D)=\sum_{D_{i}<D} g\left(D_{i}\right)
$$

so by induction

$$
\tilde{g}(D) \cdot(J \mathscr{Z})^{\left(p^{d}-1\right) /(p-1)}=0 .
$$

Hence since $\mathscr{G}(D) \cdot(J \mathscr{Z})^{p^{d}} \subseteq \tilde{G}(D)$, by (i) above, we have clearly $\mathscr{G}(D) \cdot(J \mathscr{Z})^{f}=$ 0 where

$$
f=p^{d}+\left(p^{d}-1\right) /(p-1)=\left(p^{d+1}-1\right) /(p-1)
$$

Proof of The Theorem. Let $|G|=p^{a} b$ and let $P$ be a Sylow $p$-subgroup of $G$. Then $|P|=p^{a}$ and $1 \in \mathscr{G}(P)$ so Lemma 3(ii), with $D=P$, yields

$$
(J \mathscr{Z})^{\left(p^{a+1}-1\right) /(p-1)}=0 .
$$

We close with two brief remarks. First, if $G=\langle x\rangle$ is cyclic of order $p^{a}$, then $J \mathscr{Z}=\omega(K[G])$ and the nilpotence degree of $J \mathscr{Z}$ is precisely $p^{a}$. Thus the bound given in the main theorem cannot be significantly decreased without additional assumptions on the structure of $P$.

Second, unfortunately a bound on the nilpotence degree of $J \mathscr{Z}$ does not yield a bound on the nilpotence degree of $J K[G]$. For example, let $G$ be an extra special $p$-group of order $p^{2 n+1}$. Then $\mathscr{Z}=K[Z]+\hat{Z} \cdot K[G]$ where $Z$ is the center of $G$, so $J \mathscr{Z}=\omega(K[Z])+\hat{Z} \cdot K[G]$ and $(J \mathscr{Z})^{p}=0$. On the other hand, $J K[G]=\omega(K[G])$ so the nilpotence degree of $J K[G]$ is easily seen, using [1, Theorems 3.3.6 and 11.1.9], to be $p+2 n(p-1)$. Thus the nilpotence degree of $J K[G]$ can be arbitrarily large while the nilpotence degree of $J \mathscr{Z}$ remains equal to $p$.

ACKNOWLEDGEMENT. Research partially supported by NSF Grant No. MCS 77-01775 A01. This work was done during a brief visit to the University of Essen in May 1978. I would like to thank Professor Gerhard Michler and Dr. Martin Lorenz for their kind hospitality during that stay.

\section{REFERENCES}

1. D. S. Passman, The algebraic structure of group rings, Wiley-Interscience, New York, 1977.

2. Wolfgang Willems, Über die Existenz von Blöcken, J. Algebra 53 (1978), 402-409.

Department of Mathematics, University of Wisconsin-Madison, MAdison, Wisconsin 53706 